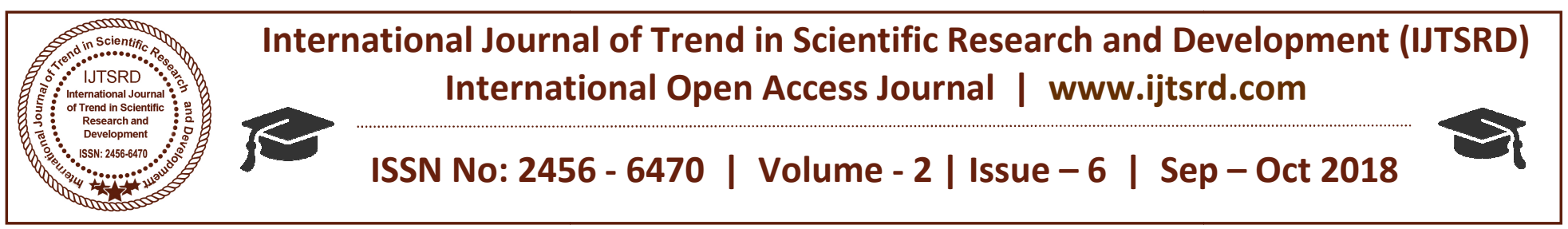

\title{
Internet of Things (IoT) Meaning, Application and Challenges
}

\author{
Ibrar Ahmed ${ }^{2}$, Shilpi ${ }^{2}$, Mohammad Amjad ${ }^{1}$ \\ ${ }^{1}$ Associate Professor, ${ }^{2}$ Student \\ Department of Computer Engineering, Jamia Millia Islamia University, New Delhi, India
}

\begin{abstract}
The idea of making self- communicating devices conceived back in 1999 however it caught attention only after the British Entrepreneur Kevin Ashton christened the term Internet of Things. Since then, many distinguished researchers and other academicians of this domain have been adding significant knowledge on the fundamental concepts of IoT in the form of extensive researches, review papers and visual presentations. Here, in this paper, we are shedding light on the core concepts of Internet of Things. We further examine the potential impact of other existing or establishing technologies on IoT. We are presenting an extensive multi-facet report on the implementation of IoT while addressing many probable challenges that may occur in future.
\end{abstract}

\section{Keyword: Internet of Things; Technologies;} Applications; Challenges

\section{INTRODUCTION}

The technological world has seen a drastic change in recent times. Among those, probably the most prominent is the advent of IoT. A scenario where network connectivity and computing capability reaches to the objects, sensors and everyday items which are otherwise not normally considered computers, enabling these devices to produce, process and consume data with minimal or no human intervention can be called 'Internet of Things'. There is, however, no single, universally accepted definition. In 1999, the concept of Internet of Things was given by a British pioneer, Kevin Ashton. In his words, IoT means a global network of things connected to RFID (radio-frequency identification). From its introduction to the present time IoT is constantly evolving.

The worldwide network of technical devices around the world is dubbed as the Internet. The internet gives an open-ended outlook for producing connectivity among many devices while the Internet of things delivers another open-ended outlook for producing connectivity as well as rooted computing capabilities among them.

Nowadays, each particular company, college, Private and Public organization even every single household is linked to one another through the internet. To be precise, every single person is connected to another through the internet. The full prospective of this interlinking is currently utilized by linking not only the people but the computing devices also. This model has been in vogue for a few years and is regarded as the internet of things. According to the definition of Internet of things, at any time a huge number of objects and things are connected to the internet and the people.

ITU-GSI [1] projected that IoT is a predominant intrinsic out of four prominent and notable research areas, they are, WSN (wireless-sensor networks), Mobile Computing Extensive computing, Internet-ofThings. IoT generally defines the method to interlink the people as well as devices to interconnect with each other and re-join back, with a very little involvement of human. Many reviewers gave their own definitions to IoT. Lee et al. [2] have observed that the IETF (Internet Engineering Task Force), world-renowned body associated to the world of internet, has termed IoT as "a wide- spanning network of interlinked things distinctively addressable based on certain communication rules".

According to Yan et al. [3], Internet-of- Things (IoT) is "a model where the devices which are capable to connect to internet, extent their limits by connecting to the things of real world such as Automobiles, TV, Refrigerator, AC, and likes. He further elaborates that the things of real world are smart enough to feel the 
other smart things nearby and they are able to communicate with each other with the help of Internet.

Vermesanet al. [4], sheds light on the possibility of bringing in the $6 \mathrm{~A}$ connectivity - that in simple words is linking individuals and things, Anytime, with Anything or Anyone from Anyplace evoking Any network or Any service. From the Chinese perspective, S. Chen et al. [5], defines IoT as "an intellectual network which links all things to the Internet with the intention of swapping knowledge and establishing connection with the knowledge- based devices by the means of reciprocal protocols". They also take IoT as an extension of already present communication among programmes and individuals by a very subtle viewpoint of "Things" for contacting. These "Things" can be seen as a grouping of Data, Services and both Software and Hardware.

Keeping in view the above definitions of IoT, we can draw our concluding definition of Internet of Things as "a model making non- living entities livable by enabling them to communicate with other devices with the help of certain network - both wired or wireless, swapping information (Read-onlyeData) among them without the need of human intervention or very subtle, if any.".

One of the most noteworthy aspect of IoT's global acceptability its permeating nature and capability to change itself as per the need or adapt the environment. With the advent of IoT, the future is set to transform into a web of interlinked entities without the discrimination of computing and Non-computing devices. The exertion of present workforce is to exploit computing power as well as the capability to connect to everything. The application of IoT is twofacet. At one hand it drives power from the field of innovation and technology at the same time takes into account the simple things of daily life. The characteristics of these things may vary to a great degree. For instance, they may be seen or unseen, Technical devices or Non-technical. Their origin can be of a public sector or a private enterprise. Their technical specification may go from simple things like chair, Pizza, and Jackets to complex devices like Printer, Walkie-Talkie and all other technically driven objects. [6] All these mentioned things can fairly establish connection among them, bringing the Sensors into play, eventually coming to the mutual aim and fulfilling the shared task. The primary significance of IoT lies in the fact of its influence on the life of its users [7].

Our goal here through this paper is to present a better and well-defined understanding of the concept of IoT by deliberating and expanding the major domains and topics associated with the application of IoT. It is well-known saying that "Mono aspect cannot explain a thing of Multi- aspect." In the same way, the Multifacet 'Internet of Things' can be defined by applying the study of various aspects. To name them, What IoT is composed of. Comparing IoT with similar domains, Technologies IoT can blend with, Platforms where IoT work, Applying IoT, Challenges of IoT.

In segment 1 , we acquaint you with the introduction of IoT, then we discuss various constituents of IoT in segment 2. Technologies which are similar to IoT are elaborated in segment 3. Technologies aiding IoT is noted in segment 4. Platforms where IoT can work is considered in segment 5. Segment 6 sees the application of IoT while segment 7 renders a considerable study of IoT's challenges while giving future discourse along with. We wind up this paper with conclusion in segment 8 and giving references in segment 9 .

\section{WHAT IOT IS COMPOSED OF}

The literal meaning of the name, Internet of things, shows the plurality it has, and indicates that it is sum of many objects, precisely Smart objects. But the list of composition does not complete here as the major constituents that form the base of IoT are: Objects, Sensors, Moving Mechanism e.g. Actuators, Transceivers, and Digital Repository.

\section{A. Objects:}

Objects coming under the realm of Internet of Things are endowed with technicalities and computing capabilities making them Smart objects. They tend to have communication interfaces enabling them contacting, sensing and exchanging information over the web. Such objects can establish contacts remotely. There is a wide variety of common objects which satisfies the criterion of smart objects. It can simply be an LED which turns on as you step in the room, A sapling which beeps when the water level goes below or a chair that weighs you as you sit.

\section{B. Sensors:}

To simply put, a sensor is a device which detects any change in the given environment. It comes first in the 
list in the application of IoT. What makes sensor a must in the implementation of IoT is its ability to gauge any variation in the given atmosphere and convert it into electric signals which then used by the other aiding devices to execute any of the task commanded. There is a wide range of sensors which can be used to implement IoT programmes. A few commonly found sensors that are widely adopted and exploited in the domain of IoT include thermometers, pressure sensors, light sensors, accelerometers, gyroscopes, motion sensors. Interaction Sensors convert a physical signal into an electrical signal that may be manipulated symbolically on a computer or device. The use of sensor varies according to the needs and place. While some works in a definite manner, others are designed to work with any given domain. As the world of technology is being flooded with IoT based devices, the stakeholders are recognizing the need of evolution in sensors particularly Multisensory integration, also known as multimodal integration devices [8].

The classification of sensors vary according to the volume of data, processing speed, single or multi domain. With their vital role in the implementation of IoT, sensors form the backbone of this technology.

\section{Moving Mechanism:}

The miniaturize technology of making microscopic machinery also known as Micro electro mechanical systems, particularly related to those with moving parts has a significant contribution in the realm of IoT. Both sensors and actuators are the product of this technology. As we already explained that sensor transforms the physical energy into electrical signals while, the prime function of the actuator is to convert the electrical signals into mechanical action. A synonym to the word "actuator" is a "mover". Actuating means inducing a movement. The first vital component of IoT i.e. sensors gather the data, that is followed by the need of processing that data with analytical approach and produce desired action. That is where the application of actuator comes into play. The issue of processing the gathered data is taken care by the actuators. That makes the duo of sensors and actuators take the first and second slot in the list of IoT implementation respectively. An actuator is simply a motor, or it can be anything that produces 'motion' or responsible for controlling some mechanism. Any machine needs some kind of energy to work, these actuators work either with electrical, hydraulic, thermal, magnetic energies. The arising need of producing more and evolved actuators is its crucial role in transforming the senses into desired action. Second reason being the deficiency in the number of existing actuators in comparison to sensors. The categorization of actuators are done on the basis of the source which they use to produce the movement. The input signals of Actuators used in the IoT is likely to be in different forms, it may be mechanical, electrical, pneumatic, or hydraulic.

\section{Transceivers:}

Transceiver is a combination of both Transmitter and Receiver. A device which is used in wireless technology such as cellular telephones, cordless telephone sets. As the IoT tends to connect everything on the network, the Transceivers have a greater role to play. In a environment of networks, a transceiver plays both a transmitter and a receiver of signals, such as analog or digital. Since the technology of Transceivers have been there for a long time, the advent of IoT has necessitated the manufacturers to design and develop modified Transceivers to exploit the ever-growing presence of IoT. As to function seamlessly in the network, every single IoT connected device needs at least one wireless transceiver chip. So, the major idea is being the introduction of 'BatteryLess Wireless Transceivers'.

\section{E. Digital Repository:}

In the IoT environment, a number of devices are simultaneously communicating with each other and generating enormous database. Which undeniably entails the need of some kind of Digital Repository for upkeeping the generated data for processing and analyzing purpose while eventually performing desired action. IoT applications are generating data deluge due to the fact of their implementation in key areas such as Health, Finance and real-time monitoring. There is constant innovation in the domain of data researchers and many game- changing technologies are set to take the world to storm such as 3D NAND, Helium drives and the futuristic DNA data storage. They seem promising as the solution of Data Saving for IoT. However, cloud storage is a good solution for the time being.

\section{COMPARING IOT WITH SIMILAR DOMAINS}

It is a general tendency of studying a domain to assess its inception, prominent features, striking functions, fundamental technicalities, visible hindrances, hurdles, and shortcomings. Apart from these, 
comparison with other similar domains is another aspect one can look at. IoT is the concept interconnecting any device with the Internet. This includes everything from Phones, Ovens, Refrigerator, Earphones, Night Lamps, wearable devices and almost anything else you can think of. The colossal network of IoT is built upon some existing technologies.

In the following para, we are projecting our subject topic i.e. Internet of Things in the light of already existing technologies.

\section{A. Internet of Things in the light of Internet:}

As the name suggests, Internet comes first in the very definition of IoT. Same is the case with its implementation. We have reviewed all the definitions and descriptions of many researchers about IoT and can conclude that Internet is the main driving agent behind the technology of IoT and its seamless functions in all the spheres. However, the simple Internet, itself is not able to connect Nonsmart devices with its ambit as it cannot sense or actuate motions in non-responding or noncomputing devices. But the IoT is endowed with additional powers of breathing lives in non- smart entities with its sensing and actuating capabilities. But it is a fact that the existence of IoT is with the existence of Internet. So we can say that the Internet makes the platform upon which Internet of Things run.

\section{B. Internet of Things in the light of Cloud computing:}

To define Cloud computing simply, it is the fetching of data and programs from a centralized store of computing resource that can be sought and used whenever desired. Cloud computing and the IoT both serve to augment the efficacy in common tasks and both have a reciprocal bonding. The IoT produces enormous data by sensing and actuating, while cloud computing delivers a route for this accumulated data to travel. The IoT produces a colosseum of Huge Data and this in turn stresses on Internet Infrastructure. Cloud computing has entered the mainstream of information technology, providing scalability in delivery of enterprise applications and Software as a Service (SaaS).

According to the arising needs, there are a number of cloud service providers have jumped to provide the hosting services of IoT. To name some, AWS, Azure,
IBM, Google Cloud, Oracle cloud. Some providers not just store the data for ready use but also provide the facility to compute the sensed data to put them into action, making Cloud Computing act as a Digital Repository for the upkeep of voluminous data and also as a computational device to aid the implementation of IoT.

\section{Internet of Things in the light of Big-data:}

The unprecedented evolution of Internet of Thing (IoT) and the fast-paced advancement of similar technologies make an extensive connectivity of "things". The deep penetration of Internet has allowed the enrollment of billions of devices into the realm of IoT. Every connected device is constantly sensing and actuating nearby devices and in turn generating a big chunk of data. The generated data is voluminous, varied and generated at different speed. That entails the need of storing processing and on demand accessing of such data. This need can be satiated with the help of acquiring the Big Data Analytic services. Some such services like SciDB, Hadoop, and TSaaaS are there that provides Big Data analytics and they are also fit for data handling requirements of Internet of Things [9]. Sensors generate the data and acts as an input for actuator that finally acts accordingly. So the Big Data has a twin role of an outcome as well as a stimulus for the effective application of Internet of Things.

\section{TECHNOLOGIES IOT CAN BLEND WITH}

To attain the maximum of any emerging domain, blending of other technologies is a must, and so is the case with IoT. Hence, below we are deliberating many present technologies which can aid the implementation of IoT.

\section{Radiofrequency identification (RFID) and Near- Field Communication (NFC):}

Radio Frequency Identification and Near Field Communication are two quite similar wireless communication technologies widely used in the application of IoT. You may use RFID or NFC technology for cashless payments, loyalty transportation, access control, Business Process Management, workforce management and E- Gov. RFID has been there for nearly two decades while NFC is comparatively a newer technology. Both the technologies are Tag- based and works on Radio waves. RFID is mainly used to transfer data. The main purpose is to identify and track automatically a 
tag attached to an object.NFC is a is a 'short-range' high frequency wireless technology that enables devices to establish a communication with each other by bringing them into proximity.

The composition of RFID involves a tag, a reader, and an antenna. The reader transmits the signals to the tag through the antenna. As an output to the input signals, the tag produces and provides desired information. A classification of the RFIDs can be into Active and Passive. The Active RFIDs are self-powered and have a range of up to 100 meters while the Passive RFIDs draw their power by the readers and have a range of up to 25 meters. Their variable ranges and power options make them an ideal technology for the proper application of IoT. The introduction of NFC was to facilitate secure data transfer. The NFC device is equipped with inbuilt Reader and Tag. This compact composition of NFC makes it capable to establish secure data connection. While RFIDs are widely used in the domain of wireless communication all over the world, the future of NFCs is bright in the time to come.

\section{A. Barcodes and Quick Response Codes (QRC):}

Barcodes are designed to contain specific product related information. It basically encodes alphanumeric characters and symbols using black and white lines, also called bars. Bar-coding is one of the AIDC (Automatic Identification and Data Collection) technologies which reduce human involvement in data entry and collection and thereby also reducing error and time. These codes contain valid and required information about the object on which they are fixed. The use of Barcoding is generally found in supermarkets for the inventory management. A successor of this technology is Quick Response Codes or QR Code. Which is more efficient and can store considerably high amount of data than to its predecessor.

Quick Response codes are Two-dimensional codes where black random-sized squares are placed on a white colored square background. The interpretation of these codes is done using scanning devices and applying some error correction methodology. The encoding modes used in these codes are generally of four types - namely; numeric, alphanumeric, binary and kanji. Another lucrative facet of these codes is their instantaneous decoding. These functions can be make use of in the implementation of IoT in identifying the things present in the web of IoT

\section{B. ZigBee:}

ZigBee or $\mathrm{X}$-Bee is another communication technology that provides short range, low power, low data rate, battery-powered applications and inexpensive substitute for IoT. It is based on the basic security framework defined in IEEE 802.15.4.Zigbee facilitates carrying out of secure communications, securing establishment and exchange of cryptographic data. ZigBee is designed in a way that its nodes can stay in sleep mode for any longer, as a result considerably increasing the battery life. The range of Zigbee is from 20 meters to 100 meters, making it ideal for Personal Area Network. In the IoT environment, where security of data is important and low transmission is not a concern, ZigBee can be best suited option for communication.

\section{6LoWPAN:}

6LoWPAN is the combined acronym of the Internet version 'IPv6' and 'Low power Wireless Personal Area Network'. The devices with limited processing ability which cannot transmit big data, can do so wirelessly using an internet protocol with the help of 6LoWPAN. IPv6 is the most recent release of Internet Protocol and it has done away the need of additional security checks with its integrated security modules which was an onerous add-on of IPv4. The introduction of 6LoWPAN was aimed to handle many other drawbacks of existing protocol by providing IPsec authentication, Flow Label field to identify packet flow for QoS handling, all-nodes multicast address, Multicast Listener Discovery (MLD).

One more important issue that it addressed is the impending shortage of network addresses. It lengthened the IP addresses from 32 bits to 128 bits. This extension corresponds to the undeniable future growth of the Internet. As the Internet of things, has in its ambit a plethora of devices which are all IP-enabled and connected to the Internet. So the basic need here is to have a technology which provides greater options to have a different identification of all the connected devices as the future of IoT is set to enroll unprecedented number of devices in its realm. With the introduction of 128 bits space for IP addresses, IPv6 has shown a revolutionary step in the communication technology. With its spacious and integrated security modules, 6LoWPAN becomes a foremost and essential technology to be blend with Internet of Things. 


\section{LTE-Advanced (LTE-A):}

LTE-Advanced is the updated version of LTE, a mobile network aimed to increase capacity and transmission speed. Also known as 4G Technology, it offers a peak data rate of at least 100 MBPS in a high speed moving conditions, and 1 GBPS in stationary conditions. The major new functionalities introduced in LTE-Advanced are Carrier Aggregation (CA), efficient use of Multi-Antenna techniques and compatibility for Relay Nodes (RN). Other technical improvements include Higher spectral efficiency, from a maximum of $16 \mathrm{bps} / \mathrm{Hz}$ in $\mathrm{R} 8$ to $30 \mathrm{bps} / \mathrm{Hz}$ in R10, Improved performance at cell edges, e.g. for DL $2 \times 2 \mathrm{MIMO}$ at least $2.40 \mathrm{bps} / \mathrm{Hz} / \mathrm{cell}$ and active management of increased number of users simultaneously. As we know IoT is loaded with so many devices at a time and every device connected to the network is generating some data every moment. For this data to be useful, movement of this data at incredibly high speed is quintessential. LTEAdvanced with its improved bandwidth and robust speed, makes it ideal network technology to be used with IoT for efficiency and timely accomplishments.

\section{E. Z-Wave:}

Z-Wave is a wireless communication protocol which is mainly used in automation of smart homes and security products. One major benefit of Z-Wave is its ability to work with any system by retrofitting standard devices. It uses low power radio signals in the $900 \mathrm{MHz}$ and automatic discovery of above 200 DPC (Device per Controller). As it is largely used in home automation, it has been optimized for reduced latency with data rate up to $100 \mathrm{KBPS}$. It automates everything from Doors to Windows to Lights and Television. Its communication system can be managed with the aid of the internet and a Z-Wave user interface. Since IoT, by definition, automates things and make them communicate, Z-Wave is deserving candidate to fall in the category of enabling technology for Internet of Things.

\section{PLATFORMS WHERE IOT WORK}

\section{A. AllJoyn:}

AllJoyn is an open source network protocol that makes the different devices interoperable. It promotes proximal network and also support cloud connectivity. The flexibility of AllJoyn can be gauged by the fact that it lets the devices sense and communicate each other even if they belong to several different domains, multiple technologies, various manufacturers. AllJoyn is based on the client-server model, the signal producing devices are known as producer (server) and the signal sensing devices are known as consumer (client). Each signal producing device is endowed with a manual which is called 'Introspection' which lets the user know of the capability of the device. AllJoyn has the flexibility to enhance its capability by bridging other protocols. There are numerous services that can be integrated with AllJoyn's core some of them are - On boarding service, Configuration service, Common Device Model service and Control Panel service.

Its major application is found in the smart home where various home devices are controlled and communicated with the common gateway of AllJoyn. With all these automated functions, we can conclude that AllJoyn is driving force of IoT.

\section{B. IoTvity:}

IoTivity is also an 'open source' platform that lets various devices connect and operate seamlessly. It was put forth by a alliance of technological companies in the communication domain, which goes by the name Open Connectivity Foundation(OCF).The underlying idea of the introduction of IoTivity is to aid and support trillions of already existing IoT devices, and paving way for the future devices which would be just double in numbers. IoTvity uses Constrained Application Protocol (CoAP) at its application layer and it has an inbuilt support for legacy protocols. The distinctive functions of IoTivityis the discovery of devices laying nearby and transmitting data from those devices. Resource scalability and added security is another factor for considering IoTvity as a qualifying platform for Internet of Things.

\section{Xively}

Xively is an IoT platform owned by Google. It has been known from various names during its period of evolution such as 'Pachube' and then 'Cosm' and eventually 'Xively'.From the services it provides, the major is being its ability to let companies connect their products, then it manages those connected devices and also manages the data of those devices while integrating them into other systems. Apart from this, it provides data services, directory services, security service in form of trust engine, and User Interface to handle the web services. Xively offers Cloud Storage Services as an add-on so it can upkeep the data processed in an IoT system. Xively uses MQTT, a technology also known as 'Publish- 
Subscribe' and is compatible with REST and Web Sockets. There are many big companies partnered with Xively such as ARM, TI, and Atmel. Some distinct factors of Xively is its capability to manage a huge number of textual transaction per day. Xively has a faster readability of product information and a swift collaboration with other systems thus automating the whole system. Xively also has the capability of real time monitoring, editing and handling the user profiles.

\section{2lemetry}

2lemetry is an IoT platform and technology company owned by Amazon Web Services (AWS) that powers the connected enterprise, people, processes, data and also IoT friendly devices. What makes it stand apart from the similar technologies is its ability of transforming raw data into ready-to-consume.

ThingFabricTM [10] IoT cloud platform of 2lemetry allows cross-communication, data storing and brokering while applying Rules and CEP Engine for preparing consumable data in real time. By providing ADI (Actionable Data Intelligence) with the aid of Predictive Computation (PC) Models and a CEP Engine, this framework also sounds reasonable to the captured data. With the services of data management, it also provides the security of data by employing many cryptographic protocols such as 'Transport Layer Security' (TLS). 2lemetryemploys different protocols for collecting data, such as RabbitMQ, WOOPSA, AMQP, HiveMQ and Solace. This platform can be integrated with Cocaine, Mendix, AWS. The major applicable domain of its function is to augment the machine to machine deployments. Commercial deployment can also exploit the usage of 2lemetry.

\section{E. HomeKit:}

HomeKit is a framework owned by Apple that allows user to configure, connect and control the appliances in user's home. There is a well-defined action for every task which can be executed with simple GUI. HomeKit can be configured in many apps to control a single device, allowing every member of a house controlling the appliances at will. It can also be configured to work with external apps for augmenting the functionality. The noticeable functions that HomeKit executes with the integration of external apps are: Finding and incorporating the appliances into the home database, allowing communication with configured devices and displaying the inputs.
HomeKit has many dedicated services to provide, some of them are HMAccessory, HMZone, HMService. The major focus of HomeKit is make the use of IoT a common household thing.

\section{F. EVRYTHNG:}

EVRYTHNG is probably the most common name as the platform for IoT. It generally is a Cloud Platform as it provides all the features of cloud system which is a pre-requisite to interconnect the devices and the Internet. It manages the real-time data and hence can go through various real-time applications simultaneously. REST API can be implemented with this technology, extending its domain of applicability. EVRYTHNG employs several Data Collection Algorithms, while the major ones being Web Sockets, Constrained Application Protocol and MQTT.

EVRYTHNG offers a robust security provision, by using twin security method - Authentication and Encryption (A\&E) employed together, allowing the connected devices and services communicate securely by PKI based on TLS certificates. In addition to that, token-based security system is also applied to prevent unauthorized access to the devices. To avoid data breach, EVRYTHNG processes the data through End to End Encryption (EEE). With the inclusion of all the necessary equipment in a single packet, EVRYTHNG becomes the worthiest platform for Internet of Things.

\section{APPLYING IOT}

In present time, IOT is found everywhere.[11] The major goal of IoT is to enable all the entities living or non-living act smart. Smartness is the key criterion for any object to work with IoT. Things which can sense, actuate some motion and send data further while doing some basic computation at their own level can be termed as smart things. Currently, there is a massive up gradation of common objects into smart objects, as a result of which IoT has taken over the technological world as well as the real world. As now IoT can be found in every domain of life, its applicability has increased drastically to every nook and corner of the physical world. A number of scientists, academicians, and other stakeholders have suggested many possible domains suitable for IoT applications. We have curated the list and subsumed the major domains of IoT applicability into twelve categories.[12] (i) Smart-cities, (ii)Smartenvironment, (iii) Smart- metering, (iv) Security \& Emergencies, (v) Retail, (vi) Logistics, 
Industrial control, (viii) Smart-Agriculture, (ix) Smart-Animal- farming,(x) Home Automation, (xi) Smart- Education and (xii) Smart Health Care. To quote Perera et al. [13], Smart Industry, Smart Environment, Smart City, Smart Wearable and Smart Home are the five major categorization of IoT solutions. The application of IoT products in its domain forms the basis of this categorization. The IoT, its products and applied method may vary in every single domain mentioned above. Sensing is not the only thing IoT is tasked with, but its operations are manifold. As per intended accomplishments and purposes, in this paper, we are listing the IoT systems into different categories. These functions can be roughly organised into the following capacities: (i) Domestic (ii) Societal

(iii) Environmental (iv) Technological (v) and Emergency and Critical Situations.

A. Domestic: Home Automation rightly falls in this category. It involves the function of sensing and automating the daily activities of home. Example may be the sensor of one's room which lets the LED know that the user is not in the room, so it may power itself off.

B. Societal: A smart IoT oriented society where everything is smart from smart traffic control system, smart transportation services, smart parking management, to smart healthcare, smart marketing and similar social services falls in this category.

C. Environmental: Smart environment where basic constituents of Environment such as the Wildlife, Marine life, Flora and Fauna are driven by IoT technologies, befits this category. An instance could be the sensor which can keep a check on the health of aquatics and prompts the authorities in real time if any mis-happening occurs.

D. Technological: Smart Industrialization, automated malls such as the Amazon stores, Smart shopping facilities and also smart security to keep all these running efficiently are the ideal components of this category.

E. Emergency and Critical Situations: Issues that are of global importance and have an impact on the very existence of mankind falls in this category. This is the domain where proper IoT implementation can bring revolutionary changes. Possible instances may include managing the natural calamities in smart way, keeping the real time eyes on water level, prompt indicators for any increased radiation, sensing toxic gas generation real fast, discovering depleting agents of ozone layer and indexing into database and augmenting border securities with the integration of IoT into the defense.

\section{CHALLENGES OF IOT}

Historically speaking, any emerging technology has both its solutions and challenges and, in our case, IoT is no different. It undoubtedly provides unprecedented easiness in human life and into the society as a whole, but there are some challenges that we find noticeable and worth mentioning side by side. The occurring challenges and their solutions may vary due to the vastness it has but here we are laying emphasis on most significant challenges that needs equal attention to derive proper productivity and aids uninterrupted application of IoT. After deeply studying the available literary, we concentrated the challenges in the system of IoT as following three categories - Technological Challenges, Societal Challenges, and Environmental Challenges.

Technological challenges begin with the scratch. IoT, primarily dealing with objects, deals with the common challenge of OOP (Object-Oriented Programming) i.e. 'Object abstraction' 'Object Encapsulation' and 'Object Inheritance' while the default Language for 'Object interaction' is another challenge. An architecture for a colossal network of trillion devices, constantly processing terabits of data is a challenge itself. Security is most vital aspect of any system and here it is again a big challenge for IoT. As increased number of devices increases the opportunity for exploiting any loopholes. Any poorly designed device may expose user data which is prone to theft by leaving data transfer inadequately protected. Another technological challenge is the abundance of identical or near identical devices. This homogeneity magnifies the potential impact of any loophole in any device as all the devices have same configurations. To deal with these challenges, a global approach of all the prominent actors coming from the IoT domain is needed.

The integrated devices may produce many privacy issues. For instance, there are many devices constantly using voice recognition and vision features, recording this personal data and processing by transferring them further, sometimes these transferences even cross physical and cultural boundaries. Any eavesdropping may cause misuse of the data and thus it is a big societal challenge that needs certain standard and well documented regulatory guidance for strengthening and standardizing these aspects. 
Environmental challenges will hold a big share in coming future. For satiating the power needs of this ever-growing network, numerous resources will be needed to put at work, stressing the power consumption scenario as well as the environmental equilibrium. The cost of implementing the IoT is another big challenge as it is projected that by 2025 , almost $35 \%$ budget resources of developing economies will go into the IoT application.

It is well-known fact that innovation and its challenges go hand in hand. But to overcome those challenges or to neutralize them to the possible extent is what sustains a technology. Future of IoT is promising and the huge number of people working behind its proper implementation is a sign of the possibility to sustain in long run.

\section{CONCLUSION}

After touching all the aspect, which is required to examine a technology, we can say Internet of Things as a domain will be dominating the technological world for decades. The aim of IoT to interconnect everything on a network is a revolutionary and it will ease the very life of people to a great extent. The scope of IoT is bottomless and it can always adapt to the situations and modify itself in accordance. The Technology of IoT is still nascent and will see considerable improvements in coming years with continuing research work in its field. The way people, organizations, and other stakeholders are incorporating IoT in their businesses; it is evident that the stage is set for IoT to envelop everything coming in its way.

In this paper, we have explained the meaning and various definitions of eminent scholars, then a detailed analysis has been done on the components of the IoT system. We further elaborated already existing technologies which can be proved a great head start for implementing IoT. The platforms of IoT are given separate attention as they are a must for setting IoT up. Lastly, we have listed all available and possible application areas for IoT with the arising challenges and some practical solutions given by prominent scholars. So, this paper can be a comprehensive and extensive reference material for all those people and researchers roped in the domain of Internet of Things.

\section{REFERENCES}

1. ITU, Internet of Things Global Standards Initiative,
T/gsi/iot/Pages/default.aspx,2015 (accessed 23.07.2018).

2. G. M. Lee, J. Park, The IoT-Concept and Problem Statement, IETF Standard draft-lee-iot problem- statement-05, (2012).

3. L. Yan, Y. Zhang, L. T. Yang, H Ning, Internet of things: from RFID to the next-generation pervasive networked systems, Auerbach Publications, (2008).

4. O. Vermesan, P. Friess, P. Guillemin, S. Gusmeroli, Sundmaeker, A. Bassi, P. Doody, Internet of things strategic research roadmap, Internet of Things: Global Technological and Societal Trends. 1 (2011) 9-52.

5. S. Chen, H. Xu, D. Liu, B. Hu, H. Wang, A Vision of IoT: Applications, Challenges, opportunities with China perspectives, Internet of Things Journal. 1(2014).

6. Dean Bubley - IoT \& Realtime Communications - IEEE IoT. (https://iot.ieee.org/newsletter/march- 2016/iotrealtime communications.html accessed on 22.07.2018)

7. J. Wan, H. Yan, H. Suo, F. Li, Advances in cyber tiphysical systems research, KSII Transactions on Internet and Information Systems. 5 (2011)1891- 1908.

8. Lizhong Wu, S. L. Oviatt, P. R. Cohen Multimodel Integration - A statistical view. IEEE Transactions on Multimedia.

9. Fog Computing: Breakthroughs in Research and Practice - Information Resources Management Association, USA. Chap-4.1 P-10.

10. Intel@ Quark ${ }^{\mathrm{TM}}$ SoC X1000 Applications Marketing Seminar. Anaheim, California, October-2014. Available online at https://www.intel.com/content/dam/www/public/ us/en/documents/training/soc-x1000-aepseminar.pdf

11. Don Norman - The Design of Everyday Things. 2014.

12. Priya Matta, Bhaskar Pant, Minit Arora - All You Want To Know About Internet of Things (IoT) (ICCCA), 2017 International Conference on. IEEE, 2017

13. C. Perera, H. Liu, S. Jayawardena, The Emerging Internet of Things Marketplace from an Industrial Perspective: A Survey, IEEE Transactions on Emerging Topics in Computing. 3 (2015) 585 -598. 